\title{
Coreference and lexical repetition: Mechanisms of discourse integration
}

\author{
KERRY LEDOUX \\ University of California, Davis, California \\ Peter C. Gordon and C. Christine Camblin \\ University of North Carolina, Chapel Hill, North Carolina \\ AND \\ TAMARA Y. SWAAB \\ University of California, Davis, California
}

\begin{abstract}
The use of repeated expressions to establish coreference allows an investigation of the relationship between basic processes of word recognition and higher level language processes that involve the integration of information into a discourse model. In two experiments on reading, we used eye tracking and event-related potentials to examine whether repeated expressions that are coreferential within a local discourse context show the kind of repetition priming that is shown in lists of words. In both experiments, the effects of lexical repetition were modulated by the effects of local discourse context that arose from manipulations of the linguistic prominence of the antecedent of a coreferentially repeated name. These results are interpreted within the context of discourse prominence theory, which suggests that processes of coreferential interpretation interact with basic mechanisms of memory integration during the construction of a model of discourse.
\end{abstract}

There are good reasons to expect that readers and listeners process words differently when they are embedded in a sentence than when those same words are embedded in a word list that lacks linguistic structure. The goal of sentence processing is to extract an integrated, coherent message from the linguistic input, a goal that is absent during the comprehension of the words in a list. In this article, we examine the possibility that processing words within a sentential context might result in effects other than, and perhaps counter to, those observed during basic lexical processing outside of a structured context.

Specifically, we address the behavioral and electrophysiological consequences of encountering lexical repetition in sentences. Studying the effects of repetition on lexical processing has proven to be a remarkably fruitful endeavor in cognitive psychology and cognitive neuroscience, providing a great deal of information about the retrieval of lexical information at different levels of processing. A fundamental effect of lexical repetition, one that plays an important role in research on word recognition and implicit memory, is repetition priming, a facilitation in the processing of a word when that word has been encountered previously.

The vast majority of studies on repetition priming have examined the processing of words presented in lists, which has been appropriate to the goals of those studies (see, e.g., Jacoby \& Dallas, 1981; Mimura, Verfaellie, \& Milberg, 1997; Scarborough, Cortese, \& Scarborough, 1977). Here, our goal is to determine whether repetition priming can be dissociated from effects that arise due to sentential context. We used eye tracking and eventrelated potentials (ERPs) during reading to examine how the structure and meaning of a sentence modulate the effects of repeating a name. We did so in order to test the hypothesis that in specific linguistic contexts, a repeated name (the function of which is ultimately to establish coreference) is, at some level, processed in the same way as a novel name. This hypothesis is derived from a model of discourse processing (Gordon \& Hendrick, 1998b) that addresses the establishment of coreference for different types of noun phrases (NPs) within and between sentences.

Two linguistic expressions are said to be coreferential if they refer to the same semantic entity; the first expression (the antecedent) introduces the entity into the discourse model, and the second expression (the anaphor) refers to it. Coreference can occur both within and between sentences and can be established by the use of full expressions (such as descriptions or names), as well as reduced expressions (such as pronouns and ellipses); it is a fundamental mechanism for making language coherent (Grosz, Joshi, \& Weinstein, 1995; Kintsch \& van Dijk, 1978).

K. Ledoux, kledoux1@jhmi.edu 
Theoretical analyses of coreference within the binding theory (Chomsky, 1981) have focused on the interaction between the syntactic position of the antecedent and the form of the anaphoric expression. Of particular relevance to this article, those analyses have explored conditions in which two expressions exhibit disjoint reference-that is, they cannot refer to the same thing. One condition under which disjoint reference is purported to arise is when the antecedent expression has a certain kind of syntactic prominence $^{1}$ in relation to an anaphoric expression that is a full NP, such as a name or a description. According to the binding theory analysis, the two occurrences of "John" in Example 1a cannot possibly refer to the same person; the syntactic prominence relation of the antecedent to the NP anaphor results in disjoint reference. On the other hand, according to the binding theory it is possible (although not necessary) that the two instances of "John" in Example 1b do refer to the same entity, because the embedding of the antecedent within the conjoined NP eliminates this syntactic prominence relation:

\section{1. a. John went to the store so that John could buy some candy. \\ b. John and Mary went to the store so that John could buy some candy.}

These principles of binding theory were developed on the basis of the metalinguistic judgments of linguists, not on the basis of the judgments of individuals who were naive to linguistic theory. Research using naive participants has yielded judgments of the acceptability of coreference that diverge sharply from those underlying the binding theory (Gordon \& Hendrick, 1997, 1998a; Keller $\&$ Asudeh, 2001). Gordon and Hendrick (1997) found that embedding an antecedent name in a conjoined NP (i.e., the contrast between $1 \mathrm{a}$ and $1 \mathrm{~b}$ ) increased the proportion of responses that deemed coreference acceptable with a subsequent repeated name. However, this effect was small, as compared with that of other factors that influenced metalinguistic judgments of coreferential acceptability. In particular, coreference in name-name sequences was deemed less acceptable than that in name-pronoun sequences, although it was deemed far more acceptable than coreference in pronoun-name sequences. This shows that pronouns provide the most natural way of establishing coreference with a previous name (at least for cases of within-sentence coreference in which the pronoun is unambiguous) but that coreference with repeated names is acceptable even when the stimulus set offers an implicit comparison with a coreferential configuration (name-pronoun sequences) that is clearly better. Gordon and Hendrick (1997) also found that the characteristics of prominence did not match those described in the binding theory (Chomsky, 1981), further indicating that characterizations of grammaticality should not rest solely on the binding theory. Finally, Gordon, Hendrick, Ledoux, and Yang (1999) reported reading-time evidence showing a strong parallel in the way in which the syntactic prominence of an antecedent affected coreferential processing of repeated names within and between sentences, a finding that suggests that restricting the analyses of these effects to the domain of a sentence (as in binding theory) misses important generalizations about coreferential processes.

The theoretical framework of Gordon and Hendrick (1998b), called discourse prominence theory, provides a synthesis of these and other findings, using formalisms adapted from model-theoretic semantics (Kamp \& Reyle, 1993). It outlines procedures for incremental processing of referential and coreferential NPs during the construction of a model of discourse and attempts to account for the interplay of syntactic prominence with different types of NPs, using the same interpretive mechanisms for coreference within sentences and between sentences in a local discourse segment. Discourse prominence theory shares basic features with other approaches that situate coreferential processing during language comprehension within the building of a model of discourse (e.g., Garnham, 2001; Johnson-Laird, 1983; Sanford \& Garrod, 1981). It differs from other approaches that have emphasized semantic factors, such as the scenario-mapping theory (Sanford \& Garrod, 1998; Sanford \& Moxey, 1995), in that it has been developed primarily to account for structural factors in coreferential processing, particularly the connection between coreferential processing and grammatical theory.

Discourse prominence theory (Gordon \& Hendrick, 1998b) specifies construction rules for interpreting different types of referential NPs in relation to the current state of the developing discourse model. The construction rule for interpreting names (and other full NPs) introduces a new semantic entity to the discourse model on which the name is predicated. In the case of a repeated name, this will result in the discourse model's containing two distinct entities with the same name, providing the basis for the intuitive sense of disjoint reference. Coreference in such cases is achieved only by additional construction rules that operate to simplify the discourse model by establishing equivalence between entities that match on their predication. The magnitude of the sense of disjoint reference and the difficulty of establishing coreference between the repeated names is determined by the characteristics of the antecedent expression. When the first occurrence of the name has prominence in the discourse model (as determined by syntactic factors related to the height of the antecedent NP in the existing syntactic structure of the sentence and, perhaps, by semantic factors), the existence of two distinct entities with the same name is subjectively apparent and impedes the establishment of coreference. Thus, a repeated name in this situation is processed for an initial period of time as if it were a new name. The additional processing that is needed to equate the two instances of the repeated name results in a repeated name penalty when the antecedent is prominent in the discourse representation (Almor, 1999; Garrod, Freudenthal, \& Boyle, 1994; Gordon, Grosz, \& Gilliom, 1993; Gordon et al., 1999; Kennison \& Gordon, 1997; Yang, Gordon, Hendrick, \& Hue, 2003). It is this prediction from discourse prominence theory that we tested by examining whether repetition priming would occur when the antecedent was linguistically prominent.

In two experiments (the first using eye tracking, and the second using ERPs), we used sentences such 
as those presented in Example 2, in which the prominence of the antecedent is manipulated by whether or not it is embedded in a conjoined NP. A number of studies have shown that this type of embedding affects the prominence of an antecedent (Albrecht \& Clifton, 1998; Gordon \& Hendrick, 1997, 1998b; Gordon et al., 1999), and the manipulation has been successfully used to study such important issues as the nature of the representation of plural entities and the processes involved in splitting a conjoined NP with singular reference (Albrecht \& Clifton, 1998; Carreiras, 1997; Kaup, Kelter, \& Habel, 2002; Koh \& Clifton, 2002; Moxey, Sanford, Sturt, \& Morrow, 2004; Sanford \& Lockhart, 1990).

\section{2. a. Prominent NP1/repeated name} At the office Daniel moved the cabinet because Daniel needed room for the desk.

b. Prominent NP1/new name At the office Daniel moved the cabinet because Robert needed room for the desk.

c. Nonprominent NP1/repeated name

At the office Daniel and Amanda moved the cabinet because Daniel needed room for the desk.

d. Nonprominent NP1/new name At the office Daniel and Amanda moved the cabinet because Robert needed room for the desk.

In these sentences, prominence of the first name in the sentence (the first instance of "Daniel" in the example) was manipulated by whether it was the sentential subject in a singular first NP (the prominent condition) or was embedded in a sentential subject consisting of a conjoined NP (the nonprominent condition); prominence is inversely related to depth of syntactic embedding (Gordon \& Hendrick, 1998b). The subject of the second clause was realized as a repeated name matching the first name in NP1 (the second "Daniel" in 2a and 2c) or as a new name ("Robert" in $2 b$ and $2 d$ ).

If repetition effects are immune to the influence of higher level processing, we might expect to see evidence of repetition priming regardless of the structural constraints of the sentence. We would thus expect to see a processing benefit for a repeated word, relative to a new word, in conditions that are otherwise identical (in $2 \mathrm{a}$ vs. $2 \mathrm{~b}$ and in $2 \mathrm{c}$ vs. $2 \mathrm{~d}$ ). We do not, however, expect this to be the case; we expect, instead, that readers will be subject to the influence of sentential context. Discourse prominence theory, and the judgment and reading time data collected in support of it, suggest a specific mechanism for this influence in sentences such as those presented in Example 2. In studies looking at explicit judgments of the acceptability of coreference with repeated names, Gordon and Hendrick (1997) showed that naive subjects consider repeated-name coreference less acceptable when NP1 is the sentential subject of a sentence (as in $2 a$ ) than when it is embedded in a sentential subject consisting of a conjoined NP (as in 2c); Gordon et al. (1999) showed parallel results with self-paced reading. Coreference will be easily and readily established using repeated names when the antecedent is not prominent in the discourse model (2c); in such sentences, the benefits of repetition and ease of integration will conspire to facilitate processing. When the antecedent is more prominent in the discourse model (2a), repeated-name coreference will be difficult. The facilitation of processing that is conferred due to repetition will be countered by a relative difficulty of integration due to structural constraints of the sentence. We might, then, predict a modulation of repetition priming in conditions in which discourse prominence inhibits the establishment of coreference.

As methods for studying online language comprehension, eye tracking and ERPs have complementary methodological strengths: Eye tracking involves the normal presentation of language stimuli and allows the speed and location of linguistic information acquisition to proceed naturally, whereas ERPs have distinctive components that have been associated with different kinds of language processes (Gordon, Camblin, \& Swaab, 2004; Kutas \& Federmeier, 1998; Sereno \& Rayner, 2003). To the extent that our manipulations have strong effects on language processing, we expected that eye-tracking and ERP results should converge. In particular, we predicted that discourse context (operationalized as the prominence of the first NP) would modulate the effects of repetition priming that might arise in the repeated name conditions. However, these methods differ in the timing in which stimulus information is available and may be differentially sensitive to some effects, as has been demonstrated by prior research in which only one of the two methods (described in more detail below) has been used. The use of both thereby provides the opportunity to obtain a more complete view of coreferential interpretation and repetition priming than could be obtained with either method alone.

\section{EXPERIMENT 1}

In our first experiment, we used eye tracking during reading to examine the factors of discourse prominence and repetition. Previous research in which eye tracking has been used during reading (Garrod et al., 1994; Gordon et al., 2004; Kennison \& Gordon, 1997) has provided information on how the prominence of a discourse referent influences subsequent coreferential interpretation of names and pronouns; however, that research did not include a lexically matched baseline, such as the newname condition used here, for assessing the presence of repetition priming. The present experiment tested whether repeated-name coreference to a prominent discourse referent would modulate repetition priming effects in eye tracking.

\section{Lexical Repetition and Eye Movements During Reading}

Surprisingly, given the large amount of research on lexical repetition in which a variety of behavioral-dependent measures has been used, relatively little research has been done on the topic with patterns of eye movements as the dependent measure. Most of the research that has been 
done has focused not necessarily on what happens when a word is encountered a second time in a given text, but instead on what happens when the same text is read a second time, sometimes with alterations of specific words, in efforts to understand the mechanisms that cause a text to be read more quickly the second time (the rereading benefit, or text repetition effect). Our experimental sentences, and their use of lexical repetition, created processing demands that were different from those created by the repetition of an entire text. First, in text repetition research, the relevant integration has been between the repeated word and earlier portions of the repeated text, not between the repeated word and the earlier instance of the repeated word, as it was here. Also, in text repetition research, the critical repeated words have had greater separation than was the case in our sentential stimuli, and those words have been surrounded by other repeated words.

However, at least one finding from text repetition studies parallels that of eye-tracking studies that focus more specifically on lexical repetition: Although both lexical repetition and synonymy have been shown to influence later eye-tracking measures associated with processes of integration, often it is only lexical repetition that influences early eye fixation measures that are typically associated with processes of lexical access (Raney, Therriault, \& Minkoff, 2000). For example, in Raney et al., lexical access alone was taken to be indicated by fixation duration when a word was fixated only once, whereas integration plus lexical access were taken to be indicated by the sum of fixation durations when a word was fixated more than once. Exact lexical repetition led to shorter fixation times for instances of both single fixations and multiple fixations, whereas synonym repetition led to shorter times only in cases of multiple fixations.

A handful of studies have used eye tracking to examine the modulation of lexical repetition effects by sentential context. In their second experiment, Traxler, Foss, Seely, Kaup, and Morris (2000) manipulated lexical repetition and sentence plausibility in sentences such as those in Example 3:

3. a. The lumberjack greeted the lumberjack early this morning.

b. The young man greeted the lumberjack early this morning.

c. The lumberjack chopped the lumberjack early this morning.

d. The young man chopped the lumberjack early this morning.

In $3 \mathrm{a}$ and $3 \mathrm{c}$, the critical word (italicized in the example) is a repetition of the sentential subject; in $3 \mathrm{~b}$ and $3 \mathrm{~d}$, it is not. In $3 \mathrm{a}$ and $3 \mathrm{~b}$, the critical word, when integrated with the preceding sentential context, creates a plausible continuation; in $3 \mathrm{c}$ and $3 \mathrm{~d}$, this word renders the sentence implausible. The effects of these two manipulations were dissociated in the eye-tracking measures; Traxler et al. reported a main effect of repetition (shorter times for repeated than for new critical words) for first-fixation duration and gaze duration but a main effect of plausibility for total read- ing time. In other words, early measures were sensitive to repetition priming, whereas later measures were sensitive to sentential context (plausibility).

Liversedge, Pickering, Clayes, and Branigan (2003) measured eye movements during the reading of adjunct phrases for which thematic role assignment (temporal or locative) was temporarily ambiguous. As is shown in Example 4, target sentences (containing the ambiguous adjunct) were preceded by a context sentence that biased a temporal or a locative thematic role assignment:

\section{4. a. Locative/locative} Context: The maid thought about where to peel/ prepare the vegetables.

Target: In fact, she peeled them in the kitchen, with great care.

b. Locative/temporal Context: The maid thought about where to peel/ prepare the vegetables.

Target: In fact, she peeled them in the morning, with great care.

c. Temporal/locative Context: The maid thought about when to peel/ prepare the vegetables.

Target: In fact, she peeled them in the kitchen, with great care.

d. Temporal/temporal

Context: The maid thought about when to peel/ prepare the vegetables.

Target: In fact, she peeled them in the morning, with great care.

Repetition of the verb across context and target sentences was also manipulated (to differentiate between a lexical locus and a discourse locus of the thematic biasing effect). A main effect of verb repetition was found for first-pass reading time of the region following the verb region (which did not include the disambiguating noun; in the in the example). Congruency effects (shorter times for congruent contexts than for incongruent ones) were found for the two regions following the disambiguating noun (the spillover region [with great] and the final region [care]). Although there was some indication that congruency influenced early processing measures for these regions (with marginally significant effects on first-pass reading time), the strongest (statistically significant) effects were on the aggregate measure of total reading time and on the later measure of rereading. These findings again suggest a dissociation between repetition priming effects (which tend to influence early eye-tracking measures) and context effects (which have a greater effect on later measures). In this experiment, as in others, these effects tended to occur downstream from the critical words themselves, in spillover regions.

Our experiment, like the two mentioned above, involved the manipulation of a lexical factor (repetition) and a context factor (coreference with a prominent antecedent). On the basis of previous results, we expected that these two factors might influence different eye-tracking measures. Early measures were expected to show evidence of repeti- 
tion priming; that is, repeated names would be read more quickly than new names when assessed by early measures of processing. On the other hand, our theoretical analysis of coreference focused on processes of integration of a word's meaning (specifically, its referent) into a discourse model. In particular, integration of a repeated name with a nonprominent antecedent was expected to be easier than integration of a repeated name with a prominent antecedent, relative to the baseline provided by the new, noncoreferential names. This ease of integration should result in shorter reading times in eye movement measures associated with integration - that is, later processing measures. These effects might be evidenced during the reading of the critical word itself or might be displaced to regions downstream in the sentence.

\section{Method}

Participants. Forty students at the University of North Carolina at Chapel Hill served as participants as part of a course requirement. One participant was dropped from the analyses and replaced by a new participant because of very long overall reading times (more than twice the group average).

Stimuli. The stimuli were adapted from those used by Swaab, Camblin, and Gordon (2004), with two important changes: (1) The pronouns were replaced by "new names," and (2) a locative phrase was placed at the start of every sentence so that the antecedent names would not occur in sentence-initial position (where position effects are strongest). Examples of the stimuli are presented in Example 2 above and in the Appendix; a full set of the materials is available from the authors. For the companion ERP experiment (Experiment 2), 160 experimental sentences were generated; of these, 40 were selected for the eye-tracking experiment. Four versions of each of the experimental sentences were constructed by manipulating two factors: prominence of NP1 and critical name type. The sentential subject consisted of either a single proper name (prominent condition) or two proper names conjoined by and (nonprominent condition). The second clause began with a temporal or a causal connective (e.g., when, after), followed by a repetition of the first-mentioned character in NP1 or a new name. The average length of the sentences was 14.56 words in the single-NP condition (range across all conditions, 10-22 words). The critical names were selected from a list of names that had been generated for previous studies (Gordon et al., 1999; Swaab et al., 2004). The length of the critical names varied between five and nine characters. Each name occurred only once within a stimulus set. Line breaks were placed in sentences in such a way that the critical name used as the subject of the second clause never appeared within two words of the beginning or the end of a line.

We conducted two offline pretests of our experimental materials; in anticipation of the ERP experiment (Experiment 2), both were conducted using the entire set of 160 experimental items. The participants were native English speakers who did not participate in the eye-tracking and ERP experiments.
Table 1

Participant Ratings of Stimuli by Experimental Condition

\begin{tabular}{lc}
\hline \multicolumn{1}{c}{ Experimental Condition } & $\begin{array}{c}\text { Mean Pretest Rating } \\
\text { (Scale of 1-5) }\end{array}$ \\
\hline Single NP1/repeated name & 3.89 \\
Conjoined NP1/repeated name & 4.28 \\
Single NP1/new name & 4.25 \\
Conjoined NP1/new name & 4.22 \\
\hline
\end{tabular}

Note-NP1, first noun phrase.

Offline test of stimulus plausibility. To ensure that the replacement of the repeated names with new names resulted in acceptable sentences, the experimental items were pretested for plausibility with 40 participants. The participants were given one of four counterbalanced stimulus sets (each of which contained 40 sentences of each of the four experimental types) and were asked to rate the sentences on a scale of 1 (does not make sense) to 5 (makes perfect sense) (see Table 1).

An ANOVA revealed a significant interaction between the two experimental factors $[F(1,39)=19.61, p<.001]$. Paired comparisons indicated that the ratings for the sentences in the single-NP1/ repeated-name condition were significantly lower than ratings for the sentences in the conjoined-NP1/repeated-name condition $[t(39)=$ $3.66, p<.001]$, the single-NP1/new-name condition $[t(39)=4.38$, $p<.001]$, and the conjoined-NP1/new-name condition $[t(39)=$ $3.05, p=.004]$. This is not surprising, given that prior judgment (Gordon \& Hendrick, 1997; Hudson, Tanenhaus, \& Dell, 1986), behavioral (Almor, 1999; Garrod et al., 1994; Gordon et al., 1993; Gordon et al., 1999; Kennison \& Gordon, 1997; Yang et al., 2003), and electrophysiological (Swaab et al., 2004) work with repeatedname coreference has shown that repeated names with prominent antecedents are difficult to process. Critically, ratings for the sentences in the remaining conditions did not differ from each other, suggesting that repeated and new names were equally plausible in the discourse context.

Offline test of the interpretation of repeated names. The results of the plausibility pretest showed lower ratings for the single-NP1/ repeated-name condition, a finding that is not surprising given previous evidence that such a configuration of referential NPs is not felicitous. Our second offline pretest used paraphrasing to test whether repeated NPs in the stimulus sentences are ultimately interpreted as coreferential (Table 2). The participants were given a sheet with experimental sentences, with a space after each sentence in which they were instructed to write a paraphrase of the sentence. The 160 experimental stimuli were divided into eight lists, each containing 20 experimental sentences. Each list was presented in four counterbalanced versions with 5 stimuli of each type, creating 32 unique questionnaires, which were completed by 32 participants. The written paraphrases for both repeated-name conditions were coded according to the attribution of the actions described in the two clauses, indicating whether the repeated name was interpreted coreferentially or as the introduction of a new character into the discourse. For some responses, interpretation of the repeated name was ambiguous, either because the two actions were not attributed to a specific

Table 2

Number and Percentage of Types of Coded Responses in the Paraphrasing Test

\begin{tabular}{|c|c|c|c|c|c|c|c|c|c|c|}
\hline \multirow[b]{4}{*}{ Condition } & & & \multicolumn{4}{|c|}{ Ambiguous } & \multirow{2}{*}{\multicolumn{4}{|c|}{ Same Person }} \\
\hline & & & \multirow{2}{*}{\multicolumn{2}{|c|}{$\begin{array}{l}\text { Repeated } \\
\text { Name }\end{array}$}} & \multirow{2}{*}{\multicolumn{2}{|c|}{$\begin{array}{l}\text { Both Actions } \\
\text { Not Included }\end{array}$}} & & & & \\
\hline & \multicolumn{2}{|c|}{ New Person } & & & & & \multicolumn{2}{|c|}{ Pronoun } & \multicolumn{2}{|c|}{ Ellipses } \\
\hline & No. & $\%$ & No. & $\%$ & No. & $\%$ & No. & $\%$ & No. & $\%$ \\
\hline Single NP & 0 & 0 & 6 & 4 & 9 & 6 & 84 & 53 & 61 & 38 \\
\hline Conjoined NP & 1 & 1 & 49 & 31 & 13 & 8 & 92 & 58 & 6 & 4 \\
\hline
\end{tabular}

Note-NP, noun phrase. 
person (e.g., The car was coming when Andrea crossed the street) or because a repeated name was used without an indication that the repeated name designated a new person, so that a pronoun could be substituted for the repeated name without introducing a semantic or grammatical anomaly (e.g., Andrea saw the car right after Andrea started to cross the street). Importantly, the overwhelming use of pronouns and ellipses in the single-NP condition indicated that the participants were interpreting these repeated names as coreferential, even though plausibility pretests had shown that this phrasing was deemed less plausible by another group of participants.

Design and Procedure. We implemented a $2 \times 2$ factorial design, with the factors of NP1 prominence (singular vs. conjoined) and critical name type (repeated vs. new) manipulated within subjects. The stimuli were counterbalanced across conditions so that no participant saw a stimulus sentence in more than one condition but, across participants, every sentence occurred in all four conditions with equal frequency. There was an initial warm-up block consisting of 16 filler trials. This was followed by five experimental blocks, each of which had eight experimental sentences (two stimuli from each of the four conditions) randomly intermixed with 14 filler trials.

The stimuli were presented on a computer screen, with eye movements measured using an EyeLink system (SensoMotoric Instruments), a head-mounted eyetracker that samples pupil location at a rate of $250 \mathrm{~Hz}$ and parses the samples into fixations and saccades. At the start of the session, the eyetracker was calibrated for each participant. Trials began with a fixation point at the location where the first word of the sentence would subsequently be presented. The fixation point served to focus the participant's attention at the correct location and also to allow the experimenter, using a second computer, to monitor the location and steadiness of the participant's gaze. The experimenter initiated the trial when the participant was appropriately fixated, causing the stimulus sentence to appear. The participant was instructed to read the sentence in a natural way, not to hurry, but not to linger excessively. He or she was asked to press the space bar when finished. Following each sentence, a true/false comprehension question appeared; the participant responded by pressing labeled keys. The experimenter's computer provided an online display of the participant's fixations. When these displays started to exhibit drift, the experimenter paused the experiment to recalibrate the tracker.

\section{Results}

Eye tracks were analyzed to provide the following standard measures of reading time (see Liversedge, Paterson, \& Pickering, 1998; Rayner, 1978, 1998): (1) first-fixation duration; (2) gaze duration (the sum of the durations of the fixations on a region of interest from the first time that region is fixated until a region outside the region of interest is fixated, provided that the eyes have not yet gone beyond the region of interest); (3) total time (the sum of all fixation durations on a region of interest); and (4) rereading duration (the difference between total reading time and gaze duration). Table 3 shows these measures for the following regions of interest: (1) the first name that appeared in the sentence, which is a possible target of rereading when trying to understand possible coreference relations; (2) the word preceding the critical name in the second clause, which provides evidence about any overall difference in difficulty due to the type of subject NP for the first clause and about possible effects of parafoveal preview of the following critical name; (3) the critical name in the second clause, which provides evidence about repetition priming and, possibly, about coreferential interpretation; and (4) the verb of the second clause (including auxiliary verbs, quantifiers, gerunds, and infinitival complements), which provides information about nonimmediate, possibly integrative effects of coreferential interpretation.

First name. There were no significant main effects or interactions on any of the reading time measures for the first name in the sentence.

Word before critical name. For first-fixation durations, times were significantly shorter in the conjoined than in the singular condition for participants, but not for items $\left[F_{1}(1,39)=5.29, p<.05 ; F_{2}(1,39)<1\right]$. Neither the effect of type of critical name nor the interaction of type of name with NP1 prominence approached significance. For gaze duration, times were again significantly shorter in the conjoined than in the singular condition for participants, but not for items $\left[F_{1}(1,39)=4.74, p<.05\right.$; $\left.F_{2}(1,39)=3.58, p<.07\right]$. Again, neither the effect of type of critical name nor the interaction of type of name with NP1 prominence approached significance. Total reading times for this region were marginally shorter in the conjoined condition than in the singular condition in the participants analysis $\left[F_{1}(1,39)=3.87, p<.06\right]$ and significantly shorter in the items analysis $\left[F_{2}(1,39)=7.3\right.$,

Table 3

Eye-Tracking Results of Experiment 1 (in Milliseconds)

\begin{tabular}{llcccc}
\hline \multirow{2}{*}{ Region of Interest } & \multicolumn{1}{c}{ Condition } & \multicolumn{4}{c}{ Measure } \\
\cline { 3 - 5 } First name & Singular/repeated & 197 & 232 & 437 & 223 \\
& Conjoined/repeated & 196 & 234 & 425 & 203 \\
& Singular/new & 193 & 226 & 411 & 204 \\
& Conjoined/new & 196 & 236 & 411 & 196 \\
Critical name minus one & Singular/repeated & 195 & 227 & 374 & 186 \\
& Conjoined/repeated & 190 & 216 & 326 & 157 \\
& Singular/new & 197 & 229 & 345 & 172 \\
& Conjoined/new & 185 & 210 & 338 & 172 \\
Critical name & Singular/repeated & 181 & 190 & 308 & 134 \\
& Conjoined/repeated & 182 & 193 & 274 & 107 \\
& Singular/new & 196 & 215 & 332 & 143 \\
Verb region & Conjoined/new & 191 & 214 & 323 & 137 \\
& Singular/repeated & & 279 & 466 & 199 \\
& Conjoined/repeated & & 263 & 407 & 158 \\
& Singular/new & & 273 & 421 & 165 \\
& Conjoined/new & & 283 & 453 & 184 \\
\hline
\end{tabular}


$p<.01]$. There was no main effect of type of critical name (repeated vs. new), nor was there a significant interaction of type of NP1 and type of critical name. For rereading, no effects approached statistical significance.

In sum, reading time measures for the word before the critical word showed some evidence of facilitated processing in the conjoined condition relative to the singular condition, although this effect was not significant in all the analyses. One possible explanation of the trend is that reading speed increases as a reader progresses further into a text (Ferreira \& Henderson, 1995) and this word is further from the beginning of the sentence in the conjoined than in the singular condition.

Critical name. For first-fixation duration, times were shorter for repeated names than for new names $\left[F_{1}(1,39)=10.56, p<.005 ; F_{2}(1,39)=10.86, p<\right.$ $.005]$, with other effects not approaching significance. Likewise for gaze duration, times were shorter for repeated names than for new names $\left[F_{1}(1,39)=22.56, p<\right.$ $\left..001 ; F_{2}(1,39)=19.09, p<.001\right]$, with other effects not approaching significance. Total reading times on the critical name were shorter for repeated names than for new names $\left[F_{1}(1,39)=17.91, p<.001 ; F_{2}(1,39)=10.07\right.$, $p<.005]$. They were also shorter for the conjoined condition than for the singular condition $\left[F_{1}(1,39)=7.30\right.$, $\left.p<.01 ; F_{2}(1,39)=5.08, p<.05\right]$. The interaction of these factors was not significant. For rereading, there was a trend toward shorter times for repeated names than for new names $\left[F_{1}(1,39)=4.28, p<.05 ; F_{2}(1,39)=3.38\right.$, $p<.08]$ and for sentences with conjoined subjects than for those with singular subjects $\left[F_{1}(1,39)=3.77, p<.06\right.$; $\left.F_{2}(1,39)=3.86, p<.06\right]$. The interaction of these factors was not significant $\left[F_{1}(1,39)=1.65, p<.21 ; F_{2}(1,39)=\right.$ $1.56, p<.25]$.

In sum, reading times for the critical name show highly reliable evidence of repetition priming (shorter times for repeated names than for new names) in measures that reflect early lexical processing (first-fixation duration and gaze duration). This difference persists, to some extent, in later measures (i.e., rereading) and comprehensive measures (i.e., total reading time) of processing. Reading times for the critical name also showed shorter total durations for sentences with plural NP1 subjects than for those with singular NP1 subjects, a pattern that is similar to what was found for the preceding word.

Verb region. First-fixation duration was not analyzed for this region because, unlike the other regions, it could consist of more than one word, which greatly limits the meaning of first-fixation duration as a measure of processing for the entire region. No significant effects were observed for this region for gaze duration measures. Total reading times for the verb region did not show significant main effects of either the type of critical name or the type of NP1 prominence. However, there was a significant crossover interaction in total reading times between these factors: For repeated names, times were shorter when the subject of the first clause was a conjoined NP than when it was a singular NP; for new names, times in these conditions showed the reverse pattern $\left[F_{1}(1,39)=5.18, p<\right.$ $\left..05 ; F_{2}(1,39)=7.22, p<.05\right]$. For rereading, there were no significant main effects, but the interaction between type of critical name and NP1 prominence was significant $\left[F_{1}(1,39)=5.53, p<.05 ; F_{2}(1,39)=4.78, p<.05\right]$.

In sum, the verb region shows evidence in a comprehensive processing measure (i.e., total reading time) and a measure of later processing (i.e., rereading) that repeatedname coreference to a prominent entity (a singular subject) creates difficulty in comprehension.

\section{Discussion}

In this first experiment, in which the participants read normally while their eye movements were monitored, we found a dissociation between effects of lexical repetition and effects of discourse context. We found evidence of repetition priming on early measures of reading at the critical word. First-fixation duration and gaze duration measures of the critical word were shorter to repeated names than to new names. In line with results from text repetition studies, the benefit conferred by repetition persisted, to some extent, in a later measure (rereading) and in a comprehensive measure (total reading time). The early emergence of this effect suggests a facilitation by repetition of processes of lexical access. The persistence of this effect suggests that repetition might also ease some aspects of discourse integration.

Critically, the discourse manipulation of the prominence of the first NP influenced the ease of establishing coreference with repeated names, as was demonstrated by the interaction of type of first NP and type of name on dependent measures reflecting later processing of the region following the critical name. When a repeated name followed a prominent antecedent, total reading times and rereading times on the verb region following the critical name were longer than those in the nonprominent condition. That this effect emerged in later durational measures suggests that it stems from integrative processes in language comprehension, not from processes of lexical access. These results provide another example of the repeated-name penalty described in previous behavioral work (Almor, 1999; Garrod et al., 1994; Gordon et al., 1993; Gordon et al., 1999; Kennison \& Gordon, 1997; Yang et al., 2003) and are, thus, consistent with the mechanisms described by the discourse prominence theory (Gordon \& Hendrick, 1998b).

\section{EXPERIMENT 2}

In the second experiment, we measured ERPs to the critical name in sentences such as those in Example 2 in order to examine the same factors of discourse prominence and repetition as in Experiment 1. Here, we will begin by reviewing the ERP effects of lexical repetition in word lists. We will then describe the handful of studies in which lexical repetition in sentence or discourse contexts has been examined.

\section{Lexical Repetition and ERPs}

The effects of repetition on ERPs to words in lists are well established, being most strongly linked to two ERP components, the N400 and the late positive com- 
plex (LPC). The N400 is a negative deflection in the ERP waveform that peaks approximately $400 \mathrm{msec}$ poststimulus-onset and is maximal over posterior electrode sites. A reduction of the amplitude of the N400 is found to words that can be easily integrated into the preceding word, sentence, or discourse context; this component is thus sensitive to processes of lexical integration (e.g., Brown \& Hagoort, 1993; Chwilla, Brown, \& Hagoort, 1995; Holcomb, 1993; Rugg, Furda, \& Lorist, 1988; van Berkum, Hagoort, \& Brown, 1999; Van Petten \& Kutas, 1991). The LPC is a positive-deflecting component that begins approximately $400 \mathrm{msec}$ post-stimulus-onset and can continue until $900 \mathrm{msec}$. It is also maximal over posterior electrode sites and is sensitive to explicit recall (Paller \& Kutas, 1992; Paller, Kutas, \& McIsaac, 1995; Rugg, 1985, 1990; Swick \& Knight, 1997).

Repetition of words in lists leads to a reduction in the amplitude of the N400 and an increase in the amplitude of the LPC. A reduction of the N400 for repeated words in lists is said to reflect the processing advantage provided by repetition. This N400 reduction has been shown to persist across lags; however, the effect is greatest at the shortest lags (Nagy \& Rugg, 1989). The increase of the LPC for repeated words in lists (relative to nonrepeated controls) has been linked to the explicit recall of the prior presentation (Paller et al., 1995).

\section{Lexical Repetition in Discourse}

A handful of studies have examined the electrophysiological response to words that are repeated within a sentence or discourse context. Even when this repetition occurs for reasons other than that of establishing coreference, the electrophysiological signature of the effects of lexical repetition in discourse contexts can differ from that for word lists. Van Petten, Kutas, Kluender, Mitchiner, and McIsaac (1991) studied lexical repetition effects in discourse, using passages taken from the Reader's Digest. As in word lists, repetitions of both content words and proper names resulted in N400 amplitude reductions. However, the LPC to these two types of words was differentially affected by repetition; whereas the LPC to proper names was more positive with repetition, it was reduced for repeated content words. Van Petten et al., interpreted these modulations of the LPC as reflecting the differential retrieval and updating demands made by content words and proper names in a discourse context. To the extent that semantic representations activated during the initial presentation of content words were still active at the time of the repetition, semantic retrieval processes were facilitated, resulting in a decrease in LPC amplitude for these words upon repetition. Because the proper names in this study referred to people who were not likely to be known to the participants, their initial presentation required little in the way of retrieval processes, since there was no prior information available to be retrieved. On subsequent presentations of the proper name, comprehension depended on the reinstatement of the prior memory representation and its updating by new discourse information. Thus, the amplitude of the LPC, as a marker of retrieval and updating, is reduced for repetitions of content words in discourse (because these words require less such processing on subsequent presentations) but increased for repeated proper names (because these words require more such processing on subsequent presentations). It is important to note that in Van Petten et al.'s study, most instances of coreference were established by the use of pronouns. It was only after a referent had been out of discourse focus for some time that a name was repeated, suggesting that these instances are better thought of as cases of reinstatement (O'Brien, Albrecht, Hakala, \& Rizzella, 1995), rather than of local coreference.

One recent study did examine lexical priming effects and discourse coreference effects during sentence processing. Anderson and Holcomb (2005) looked at the ERPs to NPs in two-sentence contexts; an example is shown in Example 5:

\section{First sentence: Kathy sat nervously in the $c a b /$ taxi on her way to the airport. \\ Second sentence: The cab came very close to hit- ting the car. \\ A cab came very close to hit- ting the car.}

Coreference was manipulated by the use in the second sentence of a definite NP (beginning with the word the) or an indefinite NP (beginning with the word $a$ ), used to refer back to a noun that had been introduced as an object in the first sentence. Priming was manipulated by the use in the second sentence of a repetition of the critical noun from the first sentence or a synonymous word. Anderson and Holcomb (2005) reported N400 priming effects at the critical word for both repetitions and synonyms; consistent with previous studies, repetition-priming effects were greater than semantic-priming effects, but neither had an effect on the LPC. However, there was no evidence of a main effect of coreference on the N400, nor was there an interaction of the two factors on this component. The absence of an interaction in this case between repetition and coreference is consistent with work related to the Gordon and Hendrick (1998b) model, because the antecedent expressions in this study were not syntactically prominent in the discourse (e.g., in a postverbal adjunct phrase).

Swaab et al. (2004) used ERPs to examine the establishment of coreference with pronouns and repeated names. They found an N400 reduction to repeated names that were preceded by a nonprominent referent, relative to those preceded by a prominent referent. Because the N400 is sensitive to difficulties in lexical integration, this difference suggests that the difficulty of achieving coreference with a name increased with the prominence of the referent.

However, in Swaab et al.'s (2004) experiment, along with those of Gordon et al. (1999), pronouns were used as a comparison for repeated names because this allowed a test of theoretical notions about the centrality of pronominal reference in discourse coherence. It is important to note, however, that names and pronouns have been shown to differ along several dimensions, such as frequency (with pronouns being more frequent in the language), length (with pronouns tending to be shorter than proper names), and 
word class (pronouns are considered closed-class words). All of these factors have been shown to affect language processing, both behaviorally and electrophysiologically (for reviews, see Hauk \& Pulvermüller, 2004; Osterhout, Allen, \& McLaughlin, 2002). For this reason Swaab et al.'s (2004) experiment provided no evidence with regard to the interaction of lexical repetition effects with discourse prominence. In the present experiment, noncoreferential new names were used to directly measure lexical repetition effects and coreferential processes during reading.

Using the conditions shown in Example 2, Experiment 2 examined the interaction of lexical repetition and discourse prominence in order to test the prediction that coreference to a prominent antecedent causes a repeated name to be processed as if it were a new name. Previous ERP research has demonstrated a reduction in the amplitude of the N400 to repeated words, in word lists and in sentence contexts; we therefore might expect to find a reduced N400 to the critical repeated name (relative to a new name) in sentences that are otherwise identical (i.e., in $2 \mathrm{a}$ vs. $2 \mathrm{~b}$ and in $2 \mathrm{c}$ vs. $2 \mathrm{~d}$ ). We predicted, however, that this repetition effect would be modulated by sentence context. Specifically, we expected that the difficulty of establishing coreference between a repeated name and a prominent antecedent would be reflected as an increase in the difficulty of integration processes in the singular-NP1/repeatedname condition (2a), relative to the conjoined-NP1/ repeated-name condition (2c), in which both repetition and ease of establishing coreference would work to reduce the amplitude of the N400. The effects of repetition on the LPC have varied in sentential contexts; difference in this experiment might be informative about differential retrieval and updating demands in these sentences.

\section{Method}

Participants. The participants were 20 right-handed native speakers of English. All had normal or corrected-to-normal vision and were recruited from the university population of the University of California at Davis. None of the participants reported neurological impairment, and none were currently taking medication. The participants gave informed consent before the experiment and were compensated with payment or with course credit.

Materials. The stimuli consisted of all of the 160 experimental items that had been pretested as described above (see Experiment 1). Each of the stimuli appeared in the same four conditions as in the previous experiment, defined by the crossing of type of subject of the initial clause (singular or conjoined) and type of subject of the second clause (repeated or new name).

Ninety filler sentences were created that also contained named characters but that had structures different from those of the experimental sentences in order to mitigate participant strategies.

Design and Procedure. Ten filler sentences formed an initial practice block, which served to familiarize the participants with the stimulus presentation and task. The 160 experimental sentences were pseudorandomly mixed with the remaining 80 filler sentences into eight subsequent blocks of 30 sentences each. The first three items and the final item in each experimental block were filler sentences. Four groupings of the experimental sentences were constructed so that a given participant read each experimental sentence once and read equal numbers of sentences in each of the conditions. Across participants, each passage occurred equally often in each condition.

Each participant was tested individually in a dimly lit, electrically shielded, sound-attenuating booth. They were seated in a comfortable chair approximately $100 \mathrm{~cm}$ from the computer screen. They were asked to silently read the sentences and to answer a true/false comprehension question after each sentence by pressing one of two buttons on a button box.

Each trial began with a fixation cross that was presented for $1,000 \mathrm{msec}$ in the center of the screen, to alert the participants to the beginning of the trial. The fixation cross was replaced by the first word of the sentence, which was replaced by subsequent words in typical rapid serial visual presentation fashion. Each sentence was presented for a duration of $300 \mathrm{msec}$ per word, with an interstimulus interval of $200 \mathrm{msec}$. Characters appeared as white letters against a dark background in 14-point Tahoma font. The first word of each sentence and all the proper names began with a capital letter; the last word was presented together with a period. During the presentation of the experimental and filler items, the participants were asked to refrain from moving their eyes and from blinking. The true/false comprehension question appeared all at once $1,000 \mathrm{msec}$ after the last word of the sentence and remained on the screen until a response was recorded. Once the participants had responded to the true/false statement, a prompt appeared on the screen, and the participants started the next trial by pressing a button. The participants were allowed a short break after each block.

EEG recording. EEG was recorded from 29 tin electrodes fitted in an elastic cap (see Figure 1), referenced to the right mastoid. Vertical eye movements were monitored by a suborbital electrode, and horizontal eye movements via left and right external canthus montages. Impedance was kept below $5 \mathrm{k} \Omega$. Prior to offline averaging, all single-trial waveforms were automatically screened for amplifier blocking, muscle artifacts, horizontal eye movements, and blinks over epochs of 1,200 msec, starting $200 \mathrm{msec}$ before the onset of the critical words. For each participant, average ERPs were computed over artifact-free trials for critical words in all four conditions. Off line, the waveforms were rereferenced to the algebraic average of both mastoids. The bandpass was $0.01-30 \mathrm{~Hz}$ at a sampling rate of $250 \mathrm{~Hz}$.

\section{Results}

ERP data were analyzed using repeated measures ANOVAs performed on the mean amplitude of the ERPs to the critical words over the 29 electrode sites in the N400 (250- to 500-msec) and LPC (500- to 700-msec) time windows (relative to a $100-\mathrm{msec}$ prestimulus baseline). In each time window, an omnibus analysis was first conducted over three independent variables, all of which were tested within subjects: NP1 prominence (with two levels: singular vs. conjoined), critical name type (with two levels: repeated vs. new), and electrode site (with 29 levels). Significant interactions were tested with subsequent contrasts, as described below. For evaluating effects with more than one degree of freedom in the numerator, the Greenhouse-Geisser correction was used to compensate for inhomogeneous variances and covariances across treatment levels (Greenhouse \& Geisser, 1959); the adjusted $p$ values are reported.

Figures 2 and 3 show the grand average ERPs in the four conditions. In Figure 2, the plots were generated to highlight the effects of the discourse manipulation, showing the grand average ERPs to the critical repeated (left panel) and new (right panel) names in the two prominence conditions. In Figure 3, the plots were generated to highlight the effects of the repetition manipulation, showing the grand average ERPs to the critical names in the prominent condition (left panel) and in the nonprominent condition (right panel).

N400 time window. An omnibus ANOVA performed on the 250- to 500-msec epoch revealed a significant in- 


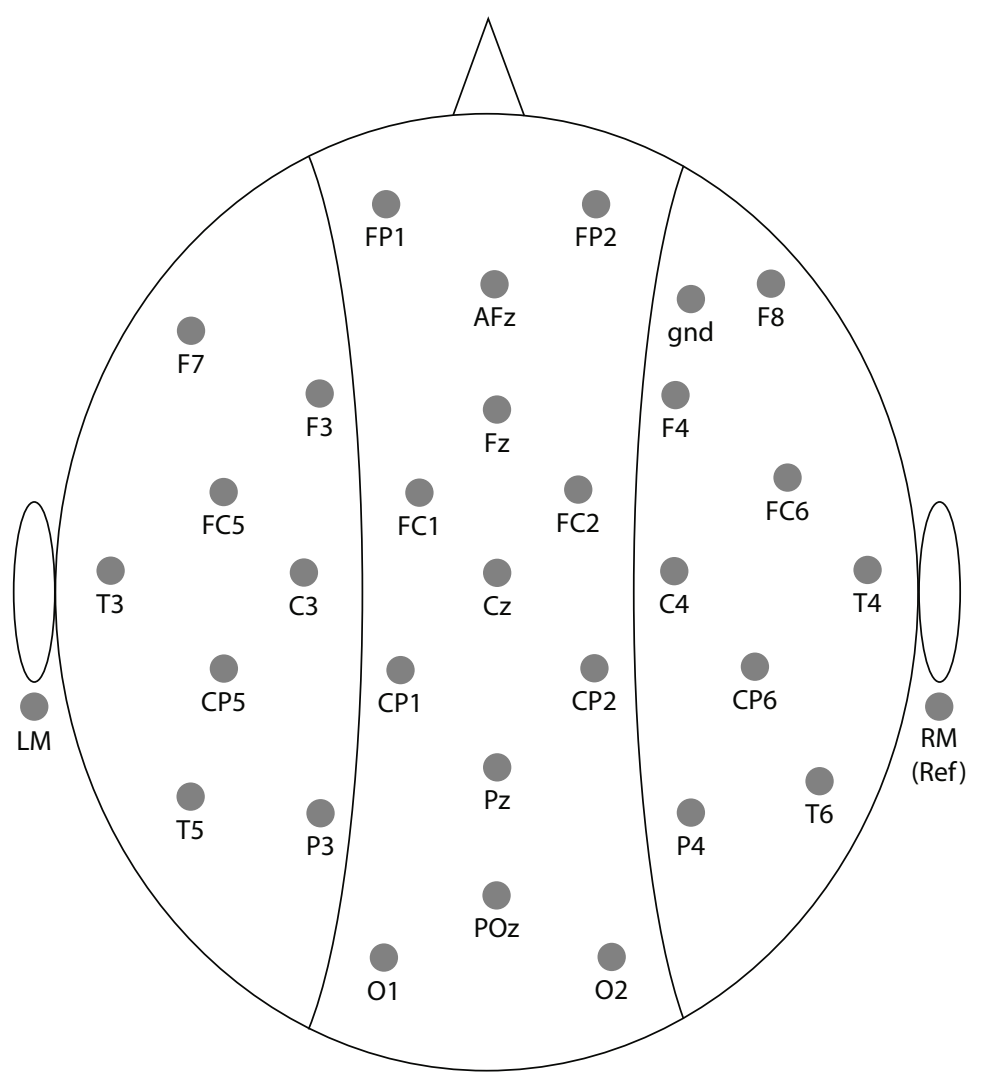

Figure 1. Configuration of the electrodes in the cap.

teraction between NP1 prominence and critical name type $[F(1,19)=5.03, p=.037]$. Paired comparisons demonstrated a significant difference for the N400 by prominence condition for the repeated names $[F(1,19)=4.79$, $p=.041]$, but not for the new names $(F<1)$. A significant difference for the $\mathrm{N} 400$ by name condition was found for the nonprominent condition $[F(1,19)=7.95, p=.011]$, but not for the prominent condition $(F<1)$.

LPC time window. As in Swaab et al.'s (2004) analysis, the interaction between discourse focus and critical name type did not reach significance in this time window $[F(1,19)=1.15, p=.338]$. However, because Swaab et al. found a significant LPC effect over posterior electrodes for repeated names, we conducted planned comparisons over these sites as well. For the repeated names, there was no significant effect of discourse focus $(F<1)$, but there was a significant interaction between this factor and electrode site $[F(28,532)=2.43, p=.04]$, demonstrating a greater positivity for repeated names following a nonprominent antecedent over posterior electrodes. There was no difference for the new names in this time window $\left(F_{\mathrm{S}}<1\right){ }^{2}$

\section{Discussion}

In this experiment, repeating a word as part of a coreferential relationship had an ERP effect similar to that seen in other situations involving repeated words-as long as the antecedent of the repeated word did not have linguistic prominence. In the nonprominent condition, a repeated coreferential name elicited a reduced N400, relative to a lexically matched new name. The LPC to these words was not influenced by repetition, a finding that differs from that of Van Petten et al. (1991). It is possible that the reading of the repeated and new names in this experiment led equally to the engagement of retrieval and updating processes. It seems, then, that processes of coreference (as studied in this experiment) and processes of reinstatement (as examined by Van Petten et al., 1991) may differentially affect the amplitude of the LPC.

Importantly, when a repeated name was coreferential with a linguistically prominent antecedent, the standard N400 repetition effect was not observed. When the subject of the first clause of the sentence consisted of a single name, the $\mathrm{N} 400$ elicited by a repeated coreferential name did not differ from that elicited by a new name. It is not the case that words in a discourse invariably benefit from repetition; this benefit may be modulated by factors that are unique to the structure of the discourse.

Repeated coreferential names that followed a prominent antecedent elicited a larger N400 than did identical repeated coreferential names that followed a nonprominent antecedent, an electrophysiological manifestation of the repeated name penalty that has been described in previous behavioral work (Almor, 1999; Garrod et al., 1994; Gordon et al., 1993; Gordon et al., 1999; Kennison \& Gordon, 1997; Yang et al., 2003). The N400 effect of discourse prominence was accompanied here (as in Swaab et al., 2004) by a difference to the repeated names in the LPC epoch. Repeated names that followed a nonpromi- 


\section{Discourse Effects}

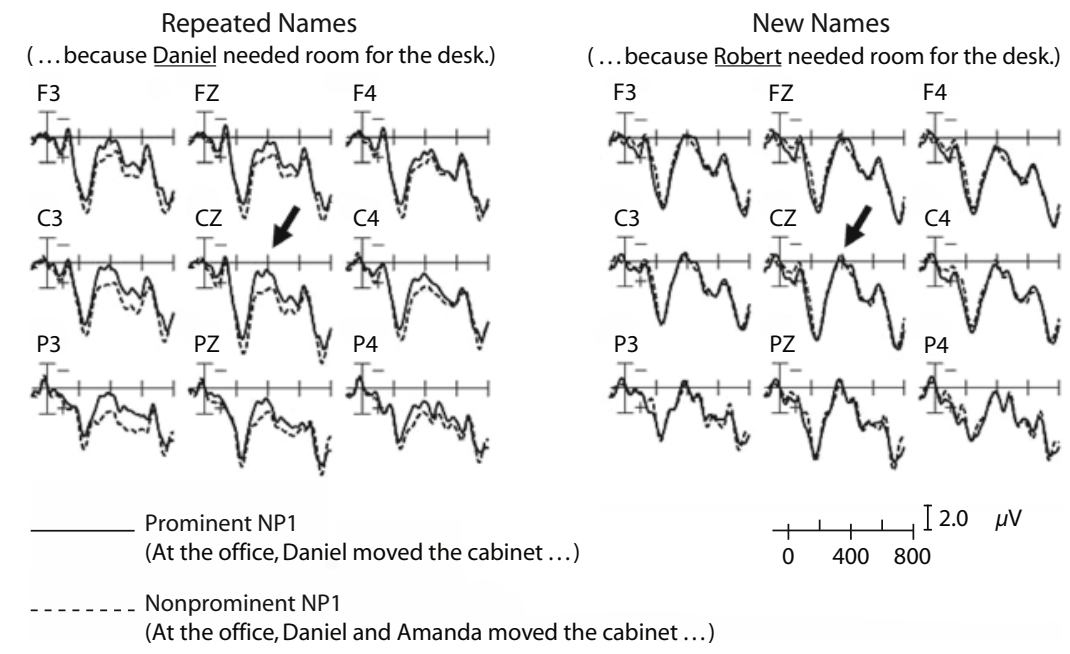

Figure 2. The effect of discourse prominence on repeated (left panel) and new (right panel) names. The ERPs are grand averages across all participants, recorded from frontal $(\mathrm{F} 3, \mathrm{Fz}, \mathrm{F} 4)$, central $(\mathrm{C} 3, \mathrm{Cz}, \mathrm{C} 4)$, and posterior $(\mathrm{P3}, \mathrm{Pz}, \mathrm{P} 4)$ sites. ERPs were time-locked to the critical name in the second clause (underlined). The arrow points to the $\mathbf{N 4 0 0}$ region on electrode $\mathrm{Cz}$.

nent antecedent elicited a greater positivity in this window than did the same repeated names when they followed a prominent antecedent. If the LPC reflects retrieval and updating processes, it seems that repeated names initiate these processes to a greater degree following a conjoined (relative to a singular) sentential subject. It seems likely that the conjoined sentential subject initially imposed a larger working memory load; the LPC difference here, then, may reflect the greater demands of reinstating and updating a larger memory load (Van Petten et al., 1991).
These findings are consistent with the mechanisms described in the Gordon and Hendrick (1998b) model. According to the discourse prominence model, repeatedname coreference to a prominent entity leads to a period of disjoint reference, wherein the discourse model contains two entities that happen to have the same name. The offline study of paraphrasing indicates that in the vast majority of cases, participants do ultimately achieve a coreferential interpretation of the repeated name in the prominent antecedent condition. The present results indicate that this

\section{Repetition Effects}

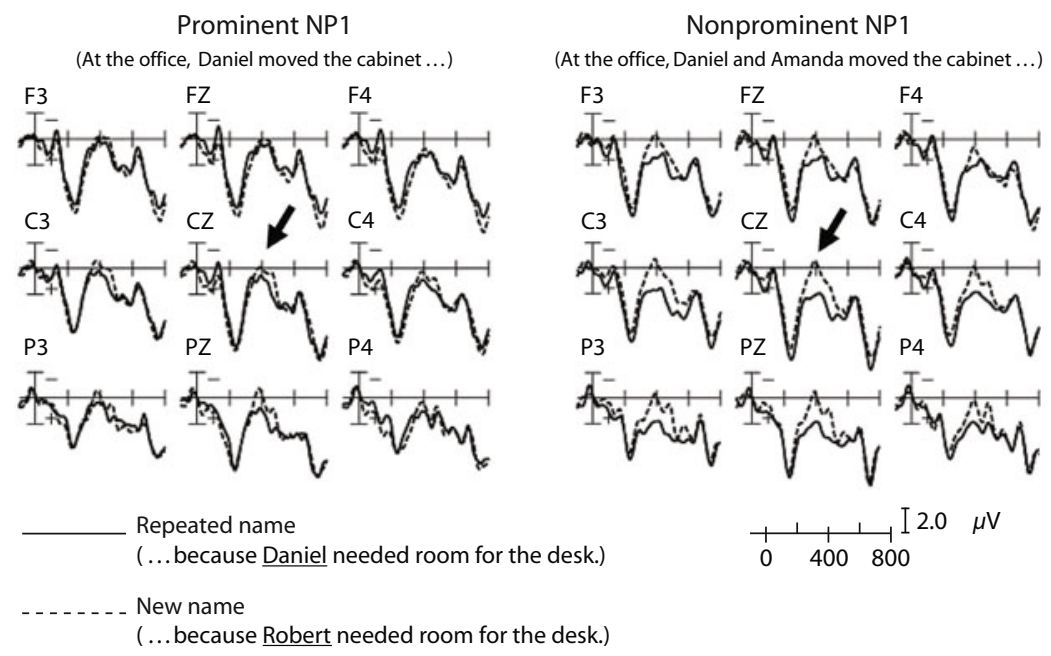

Figure 3. Repetition effect following a prominent (left panel) and a nonprominent (right panel) first noun phrase. The ERPs are grand averages across all participants, recorded from frontal $(\mathrm{F3}, \mathrm{Fz}, \mathrm{F} 4)$, central $(\mathrm{C} 3, \mathrm{Cz}, \mathrm{C} 4)$, and posterior $(\mathrm{P3}, \mathrm{Pz}, \mathrm{P4})$ sites. ERPs were time-locked to the critical name in the second clause (underlined). The arrow points to the $\mathbf{N} 400$ region on electrode $\mathrm{Cz}$. 
process is delayed past the time period measured by ERPs to the name itself.

\section{GENERAL DISCUSSION}

When presented with a task involving lists of words, participants engage in many processes that are similar to those that are engaged when words in sentences are read. Processing the words in a sentence or discourse requires the additional step of integrating lexical-semantic information into the computation of the overall meaning or context of the sentence or discourse. Coreferential interpretation of NPs is one area of discourse processing where such integration is critical. The establishment of coreference is the basis by which the characteristics and actions associated with different expressions that refer to the same entity are integrated together (Garnham, 2001; Gordon \& Hendrick, 1998b; Kintsch \& van Dijk, 1978; Sanford \& Garrod, 1981).

In two experiments, using eye tracking and ERPs, we found evidence that the sentential context in which words were repeated attenuated the magnitude of repetitionpriming effects. Effects of repetition priming were demonstrated in both experiments: In Experiment 1, durational measures of early processing (first-fixation duration and gaze duration) were shorter for repeated words than for new words, and in Experiment 2, we found a reduction of the N400 to repeated words, relative to new words. However, in both experiments, at least at some point in processing, the magnitude of these repetition-priming effects varied as a function of the type of NP that was introduced in the first clause of the sentence. In the eyetracking experiment, later processing measures (total reading time and rereading) for the region following the critical name showed repetition priming when the first NP introduced two conjoined entities to the discourse model, but not when the first NP introduced a single entity. In the ERP experiment, when the first NP introduced a single entity, repetition-priming effects were eliminated.

Both experiments also provided evidence that the processing of a coreferential repeated name was more difficult when the antecedent was prominent in the discourse representation. Readers had more difficulty integrating the two instances of a repeated name (as indexed by later processing measures in the eye-tracking experiment and by the N400 in the ERP experiment) in sentences in which the first instance was introduced as the singular subject of the first NP, relative to cases in which the first instance was embedded in a conjunctive first NP. This difficulty of integrating a coreferential repeated name with a prominent antecedent is an example of the repeated name penalty that has been described in previous behavioral work (Almor, 1999; Garrod et al., 1994; Gordon et al., 1993; Gordon et al., 1999; Kennison \& Gordon, 1997; Yang et al., 2003). We add to this body of research through the use of ERP and eye-tracking methodologies, which allowed us to more finely dissociate effects of repetition and prominence than has generally been possible using the more global behavioral measures of those studies. More important, the inclusion in the present experiments of a noncoreferential, lexically matched control for the repeated names provided a more explicit baseline for measuring repetition effects and coreferential processing than had been used in previous studies (Swaab et al., 2004), which have tended to use coreferential pronouns as a semantic control but which have not had adequate controls for lexical characteristics of the critical words.

These findings support a model of coreference such as the discourse prominence model (Gordon \& Hendrick $1998 b$ ), in which factors that are unique to the construction of a discourse model interact with general mechanisms of memory in determining the mental representation of a discourse. They show that the ease or difficulty of establishing coreference using repeated names varies depending on factors that are unique to the construction of a discourse representation. In some cases, such as those in which the antecedent is prominent in the discourse, coreference with repeated names may be exceedingly difficult; however, our offline paraphrasing study showed that readers $d o$ eventually come to take the two instances of the name to refer to the same entity. The Gordon and Hendrick (1998b) model proposes that coreferential repeated names are initially interpreted as introducing new entities to the discourse model, a suggestion that finds support in the two experiments described here. The model also proposes that at some point, further integrative processing leads to the establishment of the equivalence of the two names and to an ultimately coreferential interpretation. Whether the differences seen in the later processing measures or the amplitude of the N400 to repeated words that followed a singular antecedent reflect the positing of a new entity in the discourse model only or the additional process of establishing equivalence between two entities predicated on the same name is impossible to determine at this point.

A number of studies have compared the results from ERPs with those from eye tracking (Camblin, Gordon, \& Swaab, 2007; Deutsch \& Bentin, 2001; Gordon et al., 2004; Sereno, Rayner, \& Posner, 1998), an approach that has been advocated in the literature (Kutas \& Federmeier, 1998; Sereno \& Rayner, 2003). Our use of a subset of the ERP stimuli in the eye-tracking experiment allows such a comparison. Perhaps the most notable difference between the results of the two experiments is the locus of the majority of the significant effects. In the ERP experiment, we found evidence of repetition priming and the interaction of the repetition and discourse factors in the ERPs that were time-locked to the critical name in the second clause of the sentences. In the eye-tracking experiment, we found a different pattern of results; although we found evidence of repetition priming on the critical word itself, evidence of the interaction of this factor with discourse prominence was displaced to the region following the critical word. In addition, in the eye-tracking experiment, the effect of repetition was seen to influence (primarily) early processing measures, whereas the discourse effect was seen to influence later processing measures; in the ERP experiment, both of these factors influenced the same component (and thus, a similar time course of expression). These differences may result from basic methodological differences between the ERP and the eye-tracking paradigms. In our 
ERP experiment, as in many such studies in which reading has been examined, we used rapid serial visual presentation with a relatively slow presentation rate of $500 \mathrm{msec}$ per word to allow better resolution of the ERP components of interest. However, this means that the reader is made to look at each word longer than the typical fixation rate during natural reading of approximately $250 \mathrm{msec}$. This is not the case in the eye-tracking experiment, in which readers have control of where they are looking and for how long. The prolonged presentation duration in the ERP paradigm may give the reader enough time to engage in processes (such as those of coreferential integration) that would normally be extended over a region of several words in natural reading. Indeed, recent models of eye movements during reading describe processing as distributed in nature, in that a given fixation on a word might reflect the simultaneous contributions from the processing of the previous, current, and subsequent words (Kliegl, Nuthmann, \& Engbert, 2006). In the eye-tracking results, we may be seeing processes of lexical access (priming) being localized to the critical word itself, but processes of sentential integration (as influenced by discourse prominence) spread over a number of words.

In summary, we measured eye movements and ERPs to coreferential repeated names (and noncoreferential new name controls) in order to examine mechanisms of discourse integration. Processing the coreferential relationship between two expressions requires the establishment and maintenance of a representation of the information conveyed by the antecedent expression, followed by retrieval of that information on the basis of cues in the coreferential expression. These processes of representation and retrieval are strongly influenced by syntactic and discourse structure.

\section{AUTHOR NOTE}

This research was supported by NIMH Grant RO1 MH066271 to P.C.G. and T.Y.S. We thank Scott Hajek and Stephanie Moser for assistance with data collection. Correspondence concerning this article should be addressed to K. Ledoux, Cognitive Neurology/Neuropsychology, Johns Hopkins University, 1629 Thames St., Suite 350, Baltimore, MD 21231 (e-mail: kledoux1@jhmi.edu).

\section{REFERENCES}

Albrecht, J. E., \& Clifton, C., JR. (1998). Accessing singular antecedents in conjoined phrases. Memory \& Cognition, 26, 599-610.

Almor, A. (1999). Noun-phrase anaphora and focus: The informational load hypothesis. Psychological Review, 106, 748-765.

Anderson, J. E., \& Holcomb, P. J. (2005). An electrophysiological investigation of the effects of coreference on word repetition and synonymy. Brain \& Language, 94, 200-216.

Brown, C. M., \& Hagoort, P. (1993). The processing nature of the N400: Evidence from masked priming. Journal of Cognitive Neuroscience, 5, 34-44.

Camblin, C. C., Gordon, P. C., \& SwaAb, T. Y. (2007). The interplay of discourse congruence and lexical association during sentence processing: Evidence from ERPs and eye tracking. Journal of Memory \& Language, 56, 103-128.

Carreiras, M. (1997). Plural pronouns and the representation of their antecedents. European Journal of Cognitive Psychology, 9, 53-87.

Chомsкy, N. (1981). Lectures on government and binding. Dordrecht: Foris.

Chwilla, D. J., Brown, C. M., \& Hagoort, P. (1995). The N400 as a function of the level of processing. Psychophysiology, 32, 274-285.

Deutsch, A., \& Bentin, S. (2001). Syntactic and semantic factors in processing gender agreement in Hebrew: Evidence from ERPs and eye movements. Journal of Memory \& Language, 45, 200-224.

Ferreira, F., \& Henderson, J. M. (1995). Reading processes during syntactic analysis and reanalysis. In J. M. Henderson, M. Singer, \& F. Ferreira (Eds.), Reading and language processing (pp. 119-147). Mahwah, NJ: Erlbaum.

GARNHAM, A. (2001). Mental models and the interpretation of anaphora. Hove, U.K.: Psychology Press.

Garrod, S., Freudenthal, D., \& Boyle, E. A. (1994). The role of different types of anaphor in the on-line resolution of sentences in a discourse. Journal of Memory \& Language, 33, 39-68.

Gordon, P. C., Camblin, C. C., \& SwaAb, T. Y. (2004). On-line measures of coreferential processing. In M. Carreiras \& C. Clifton (Eds.), The on-line study of sentence comprehension: Eyetracking, ERPs, and beyond (pp. 139-150). New York: Psychology Press.

Gordon, P. C., Grosz, B. J., \& Gilliom, L. A. (1993). Pronouns, names, and the centering of attention in discourse. Cognitive Science, 17, 311-347.

Gordon, P. C., \& HENDRICK, R. (1997). Intuitive knowledge of linguistic coreference. Cognition, 62, 325-370.

GoRdon, P. C., \& HENDRICK, R. (1998a). Dimensions of grammatical coreference. In M. A. Gernsbacher \& S. J. Derry (Eds.), Proceedings of the Twentieth Annual Conference of the Cognitive Science Society (pp. 424-429). Hillsdale, NJ: Erlbaum.

GoRdon, P. C., \& HENDRICK, R. (1998b). The representation and processing of coreference in discourse. Cognitive Science, 22, 389-424.

Gordon, P. C., Hendrick, R., Ledoux, K., \& Yang, C. L. (1999). Processing of reference and the structure of language: An analysis of complex noun phrases. Language \& Cognitive Processes, 14, 353-379.

Greenhouse, S. W., \& GeIsSER, S. (1959). On methods in the analysis of profile data. Psychometrika, 24, 95-112.

Grosz, B. J., Joshi, A. K., \& Weinstein, S. (1995). Centering: A framework for modeling the local coherence of discourse. Computational Linguistics, 21, 203-225.

Hauk, O., \& Pulvermüller, F. (2004). Effects of word length and frequency on the human event-related potential. Clinical Neurophysiology, 115, 1090-1103.

Holсомв, P. J. (1993). Semantic priming and stimulus degradation: Implications for the role of the $\mathrm{N} 400$ in language processing. Psychophysiology, 30, 47-61.

Hudson, S. B., Tanenhaus, M. K., \& Dell, G. S. (1986). The effect of the discourse center on the local coherence of a discourse. In Proceedings of the 8th Annual Conference of the Cognitive Science Society (pp. 96-101). Hillsdale, NJ: Erlbaum.

JACOBY, L. L., \& DALLAS, M. (1981). On the relationship between autobiographical memory and perceptual learning. Journal of Experimental Psychology: General, 110, 306-340.

JOHNSON-LAIRD, P. N. (1983). Mental models: Toward a cognitive science of language, inference, and consciousness. Cambridge, MA: Harvard University Press.

Kamp, H., \& Reyle, U. (1993). From discourse to logic: Introduction to model theoretic semantics of natural language, formal logic, and discourse representation theory. Dordrecht: Kluwer.

Kaup, B., Kelter, S., \& Habel, C. (2002). Representing referents of plural expressions and resolving plural anaphors. Language \& Cognitive Processes, 17, 405-450.

Keller, F., \& Asudeh, A. (2001). Constraints on linguistic coreference: Structural vs. pragmatic factors. In J. D. Moore \& K. Stenning (Eds.), Proceedings of the 23rd Annual Conference of the Cognitive Science Society (pp. 483-488). Mahwah, NJ: Erlbaum.

Kennison, S. M., \& Gordon, P. C. (1997). Comprehending referential expressions during reading: Evidence from eye tracking. Discourse Processes, 24, 229-252.

KInTSCH, W., \& VAN DiJK, T. A. (1978). Toward a model of text comprehension and production. Psychological Review, 85, 363-394.

Kliegl, R., Nuthmann, A., \& Engbert, R. (2006). Tracking the mind during reading: The influence of past, present, and future words on fixation durations. Journal of Experimental Psychology: General, 135, 12-35.

KoH, S., \& Clifton, C. (2002). Resolution of the antecedent of a plural pronoun: Ontological categories and predicate symmetry. Journal of Memory \& Language, 46, 830-844.

Kutas, M., \& Federmeier, K. D. (1998). Minding the body. Psychophysiology, 35, 135-150. 
Liversedge, S. P., Paterson, K. B., \& Pickering, M. J. (1998). Eye movements and measures of reading time. In G. Underwood (Ed.), Eye guidance in reading and scene perception (pp. 55-75). Amsterdam: Elsevier.

Liversedge, S. P., Pickering, M. J., Clayes, E. L., \& Branigan, H. P. (2003). Thematic processing of adjuncts: Evidence from an eye-tracking experiment. Psychonomic Bulletin \& Review, 10, 667-675.

Mimura, M., Verfaellie, M., \& Milberg, W. P. (1997). Repetition priming in an auditory lexical decision task: Effects of lexical status. Memory \& Cognition, 25, 819-825.

Moxey, L. M., Sanford, A. J., Sturt, P., \& Morrow, L. I. (2004). Constraints on the formation of plural reference objects: The influence of role, conjunction, and type of description. Journal of Memory \& Language, 51, 346-364.

NAGY, M. E., \& RUGG, M. D. (1989). Modulation of event-related potentials by word repetition: The effects of inter-item lag. Psychophysiology, 26, 431-436.

O’Brien, E. J., Albrecht, J. E., Hakala, C. M., \& Rizzella, M. L. (1995). Activation and suppression of antecedents during reinstatement. Journal of Experimental Psychology: Learning, Memory, \& Cognition, 21, 626-634.

Osterhout, L., Allen, M., \& Mclaughlin, J. (2002). Words in the brain: Lexical determinants of word-induced brain activity. Journal of Neurolinguistics, 15, 171-187.

PAller, K. A., \& KutAs, M. (1992). Brain potentials during memory retrieval provide neurophysiological support for the distinction between conscious recollection and priming. Journal of Cognitive Neuroscience, 4, 375-391.

Paller, K. A., Kutas, M., \& McIsaac, H. K. (1995). Monitoring conscious recollection via the electrical activity of the brain. Psychological Science, 6, 107-111.

Raney, G. E., Therriault, D. J., \& Minkoff, S. R. B. (2000). Repetition effects from paraphrased text: Evidence for an integrated representation model of text representation. Discourse Processes, 29 61-81.

RAYNER, K. (1978). Eye movements in reading and information processing. Psychological Bulletin, 85, 618-660.

RAYNER, K. (1998). Eye movements in reading and information processing: 20 years of research. Psychological Bulletin, 124, 372-422.

RugG, M. D. (1985). The effects of semantic priming and word repetition on event-related potentials. Psychophysiology, 22, 642-647.

RuGG, M. D. (1990). Event-related brain potentials dissociate repetition effects of high- and low-frequency words. Memory \& Cognition, 18, 367-379.

RugG, M. D., Furda, J., \& Lorist, M. (1988). The effects of task on the modulation of event-related potentials by word repetition. Psychophysiology, 25, 55-63.

SANFORD, A. J., \& GARROD, S. C. (1981). Understanding written language: Explorations of comprehension beyond the sentence. New York: Wiley.

SANFORD, A. J., \& GARROD, S. C. (1998). The role of scenario mapping in text comprehension. Discourse Processes, 26, 159-190.

SANFORD, A. J., \& Lockhart, F. (1990). Description types and method of conjoining as factors influencing plural anaphora: A continuation study of focus. Journal of Semantics, 7, 365-378.

SANFord, A. J., \& Moxey, L. M. (1995). Notes on plural reference and the scenario-mapping principle in comprehension. In C. Habel $\&$ G. Rickheit (Eds.), Focus and coherence in discourse processing (pp. 18-34). Berlin: de Gruyter.

Scarborough, D. L., Cortese, C., \& Scarborough, H. S. (1977). Frequency and repetition effects in lexical memory. Journal of Experimental Psychology: Human Perception \& Performance, 3, 1-17.

Sereno, S. C., \& Rayner, K. (2003). Measuring word recognition in reading: Eye movements and event-related potentials. Trends in $\mathrm{Cog}$ nitive Sciences, 7, 489-493.

Sereno, S. C., Rayner, K., \& Posner, M. I. (1998). Establishing a time-line of word recognition: Evidence from eye movements and event-related potentials. NeuroReport, 9, 2195-2200.

SwaAb, T. Y., Camblin, C. C., \& Gordon, P. C. (2004). Reversed lexical repetition effects in language processing. Journal of Cognitive Neuroscience, 16, 715-726.

SwICK, D., \& KNIGHT, R. T. (1997). Event-related potentials differentiate the effects of aging on word and nonword repetition in explicit and implicit memory tasks. Journal of Experimental Psychology: Learning, Memory, \& Cognition, 23, 123-142.

Traxler, M. J., Foss, D. J., Seely, R. E., Kaup, B., \& Morris, R. K. (2000). Priming in sentence processing: Intralexical spreading activation, schemas, and situation models. Journal of Psycholinguistic Research, 29, 581-594.

van Berkum, J. J. A., Hagoort, P., \& Brown, C. M. (1999). Semantic integration in sentences and discourse: Evidence from the N400. Journal of Cognitive Neuroscience, 11, 657-671.

Van Petten, C., \& Kutas, M. (1991). Influences of semantic and syntactic context on open- and closed-class words. Memory \& Cognition, 19, 95-112.

Van Petten, C., Kutas, M., Kluender, R., Mitchiner, M., \& McIsAAC, H. (1991). Fractionating the word repetition effect with eventrelated potentials. Journal of Cognitive Neuroscience, 3, 131-150.

Yang, C. L., Gordon, P. C., Hendrick, R., \& Hue, C. W. (2003). Constraining the comprehension of pronominal expressions in Chinese. Cognition, 86, 283-315.

\section{NOTES}

1. The relation is called a $c$-command and is defined as follows: $\alpha$ c-commands $\beta$ if and only if the first branching node above $\alpha$ contains $\beta$ (see Gordon \& Hendrick, 1997, 1998b, for a discussion and an alternative formulation of the critical syntactic relation).

2. Because of the presentation rate used in the experiment, the signal to the critical word in the LPC time window overlaps with that of the following word. There are two reasons to believe that the LPC effects we see were due to processing of the critical word. First, the word following the critical word was the same in all the experimental conditions; differences in processing this word were thus unlikely to arise. Second, if we time-locked the signal to the following word, we did not see significant differences in the ERP. 


\section{Twenty Examples of the Experimental Stimuli in the Four Conditions Used in the Experiments}

1. Last week Nicole (and Duncan) joined protests against the tuition hike because Nicole/Bonnie could not afford the new rate.

2. Understandably Andre (and Molly) left the party early after Andre/Devin made a rude comment at dinner.

3. At the mall Gwendolyn (and Frederick) shopped for tents before Gwendolyn/Priscilla went camping.

4. Out in the field Jeffrey (and Serena) set up the telescope before Jeffrey/Anthony started looking at the moon.

5. Yesterday Patricia (and Clifford) bought a new sweater because Patricia/Jennifer had spilled red wine all over the old one.

6. With reluctance Dylan (and Edith) washed the dishes while Dylan/Wayne talked about the upcoming election.

7. Despite the distance Sheila (and Eugene) looked for a house near the college after Sheila/Deidre was mugged downtown.

8. Last Friday Kevin (and Doris) left work early after Kevin/Scott completed work on the project.

9. Based on the schedule Alexis (and Calvin) wrote the lyrics to the song before Alexis/Sandra composed the music.

10. In spite of the rain Jared (and Norma) enjoyed the concert at which Jared/Damon met the band.

11. During the night Leila (and Bruce) called home because Leila/Dinah had crashed the car.

12. If asked Miguel (and Helene) always sang at parties when Miguel/Emmett rolled out the piano.

13. With great care Felicia (and Charles) painted the living room while Felicia/Dolores was on vacation from work.

14. Quite spontaneously Edwin (and Shari) got married when Edwin/Oscar lived in Washington.

15. Despite the weather Janine (and Nathan) went to the beach when Janine/Violet rented a house for a week.

16. After the game Irving (and Gloria) got pizza because Irving/Norris doesn't like Chinese food.

17. Until last year Cynthia (and Vincent) often bought books online until Cynthia/Natalie thought of borrowing from the library.

18. Eventually Kenny (and Rosie) stopped playing golf because Kenny/Aaron said it was a waste of time.

19. Fearfully Ashley (and Roland) gasped in horror before Ashley/Daphne discovered that the horrible scars were just make-up.

20. Every week Albert (and Hannah) went to the theater because Albert/Oliver gave free acting lessons.

(Manuscript received September 1, 2005;

revision accepted for publication March 20, 2006.) 